\title{
Psychological distress and coping in military cadre candidates
}

\author{
This article was published in the following Dove Press journal: \\ Neuropsychiatric Disease and Treatment \\ 29 August 2016 \\ Number of times this article has been viewed
}

\author{
Can Nakkas' \\ Hubert Annen' \\ Serge Brand ${ }^{2,3}$ \\ 'Department of Military Psychology \\ Studies, Military Academy at ETH \\ Zurich, Zurich, ${ }^{2}$ Psychiatric Clinics of \\ the University of Basel, Center for \\ Affective, Stress and Sleep Disorders, \\ ${ }^{3}$ Department of Sport, Exercise \\ and Health, Sport Science Section, \\ University of Basel, Basel, Switzerland
}

Correspondence: Can Nakkas Ackersteinstrasse 130, Zurich $\mathrm{CH}-8049$, Switzerland Email c.nakkas@gmail.com
Background: Soldiers must cope with stressors during both military operations and training if they are to accomplish their missions successfully and stay mentally stable. This holds true particularly for military superiors, as they bear greater responsibilities and must meet greater demands during both deployment and training. Accordingly, in the present study, we investigated whether recruits chosen for further promotion at the end of basic training differed with regard to psychological distress and coping strategies from those not chosen for promotion, and whether recruits' coping styles and distress levels were associated.

Methods: A total of 675 Swiss recruits took part in the study. At the beginning of basic training, recruits filled out self-rating questionnaires covering demographic data, psychological distress (depression, somatization, anxiety, interpersonal sensitivity, and hostility), and coping styles. Results were compared between those recruits who received a recommendation for further promotion at the end of basic training and those who did not.

Results: Recruits selected for promotion had lower scores for depressive symptoms and hostility, engaged more in active coping, and considered their coping to be more effective. Dysfunctional and functional coping were associated with higher and lower distress levels, respectively.

Conclusion: Recruits recommended for promotion exhibited less psychological distress during basic training and exhibited a socially more conducive profile of distress. They also endorsed more efficient and more prosocial coping strategies than those recruits not recommended for promotion. These cognitive-emotional features not only contribute to resilience but are also consistent with leadership research, indicating the importance of emotional stability and prosocial behavior in successful leaders.

Keywords: military, training, stress, recruits, coping, leadership

\section{Introduction}

By definition, soldiers do dangerous and stressful work, and military personnel have an elevated risk of suffering from stress-related disorders following military deployment. ${ }^{1}$ The risks of developing other psychological disorders such as depression, anxiety, and alcohol abuse are also higher. ${ }^{2}$ Although the military operates under different conditions in peace and war, military training in peacetime is nonetheless intended to provide preparation for combat situations. Its goal is to enable service personnel to perform under adverse conditions that offer little control, as a soldier's performance in military operations is mission-critical and allows for little leeway. ${ }^{3}$

It thus comes as no surprise that basic military training itself has also been shown to be a biopsychosocial stressor, resulting in elevated cortisol levels ${ }^{4-7}$ and elevated selfreported psychological distress. ${ }^{8-10}$ Although most recruits adapt to basic training, $, 9,11$ the literature also includes reports of fluctuating levels of stress and negative mood over time. ${ }^{8,9,12}$ 
Research indicates that stress resistance is a predictor for successful completion of demanding training courses such as basic military training. High levels of hardiness, for example, predict successful graduation from officer training ${ }^{13}$ and firefighter training ${ }^{14}$ and reduce the risk of developing psychological problems during military training. ${ }^{15}$ Coping strategies have also been shown to reduce the negative effects of stress on psychological health. Although emotion-oriented and avoidance coping is generally related to negative health outcomes, problem- and approach-oriented coping is associated with positive health outcomes. ${ }^{16,17}$ In a military setting, Mikulincer and Florian ${ }^{18}$ found that recruits who engaged in emotion-oriented coping considered basic training as threatening, whereas recruits who engaged in problem-oriented coping considered it as a challenge. In addition, the more effective a recruit appraised his coping in basic training, the more likely he was to engage in problem-oriented coping, and the less likely he was to engage in emotion-oriented coping. In the present study, we thus also assessed the coping styles of recruits during basic training.

Why is it important to know how recruits experience stress during basic training and how they cope with it? If recruits in general need to be able to withstand the hardships of military training, this holds true particularly for the prospective leaders among them. Junior military cadre, ie, squad leaders and platoon leaders, must meet high demands. On the one hand, they are accountable for implementing basic training and must answer to their superior officers, on the other they are their subordinates' first point of contact in disciplinary and personal matters. This combination of technical and social demands is a stressor in itself and requires that they exhibit both task orientation and people orientation, the two main behavioral dimensions in classical leadership theory. ${ }^{19}$

Research on leadership emergence, ie, the psychological preconditions that predict the emergence of leadership in groups, also points toward stress resistance as a distinguishing factor. ${ }^{20}$ Studies on vertebrates have shown that in contrast to the "reactive" phenotype, "proactive" animals exhibit endocrine and behavioral responses to stress that promote leadership emergence, eg, lower levels of hypothalamicpituitary-adrenocortical axis hormones, higher adrenaline levels, and higher likelihood of initiation of and adherence to social interaction. ${ }^{21}$ This coincides with research on leadership emergence in humans: emergent leaders adjust better to stressful situations ${ }^{22}$ and exhibit higher levels of agreeableness and openness to new experiences. ${ }^{23,24}$ In addition, elevated hypothalamic-pituitary-adrenocortical axis responses are often associated with higher aggression. ${ }^{25,26}$ We thus anticipated that recruits who reported less psychosocial stress and more active and problem-oriented coping styles would be perceived as promising cadre by their superiors. In contrast, leadership stress has been shown to have negative effects on performance both in civilian ${ }^{27}$ and military settings. Fiedler et $\mathrm{al}^{28}$ showed in an experiment with Reserve Officer Training Course cadets that social stress negatively affected leadership decisions. Conversely, the military cadre's own stress resistance acted as a top-down stress buffer for their subordinates' stress levels, as soldiers who attributed high leadership skills to their superiors suffered from less stress. ${ }^{29-31}$ West Point cadets exhibited both effective problem- and emotion-oriented coping (eg, using social support and engaging in physical activity, humor, and rationalization) during basic training, ${ }^{32}$ although rank and experience also appear to factor into one's choice of coping style. Compared to soldiers, noncommissioned officers (NCOs) and officers reported less passive coping, which is associated with lower psychological well-being ${ }^{33}$ and characterized by avoidance, withdrawal, and wishful thinking (eg, complaining about the situation to ventilate feelings, get sympathy, or elicit help; withdrawing from social activities; and relying on medication). ${ }^{34}$ Results from a civilian sample also show that individuals were evaluated as more effective leaders by their subordinates if they exhibited active coping, ${ }^{35}$ which is characterized by using one's own resources to deal with a problematic situation, improving the nature of the stressful situation, or modifying one's emotional and/or cognitive reactions (eg, solving problems, reframing the meaning of the problem, and seeking information). ${ }^{36}$ Recruits who engaged in mere emotion-oriented coping, however, were rated as less qualified to become officers or NCOs by their peers. ${ }^{18}$ Finally, soft factors such as social judgment skills and agreeableness were shown to be predictive of military leadership performance in West Point cadets. ${ }^{37}$

Taken together, from previous research, we know that basic military training is a psychological stressor and that stress resistance as well as active and problem-oriented coping are associated with better mental health outcomes, successful completion of military training, and stronger, more prosocial leadership qualities. We do not know, however, whether future officers and NCOs suffer from less stress during basic training than their comrades who do not get promoted. The aim of the present study was therefore to assess psychological distress and coping strategies among recruits during the first month of basic training and to compare these results between recruits who were recommended for promotion at the end of basic training and those who were not. Accordingly, the following three hypotheses were 
formulated: First, following Bartone,${ }^{29}$ Bliese, ${ }^{30}$ and Castro, ${ }^{31}$ we expected lower psychological distress in recruits who went on to receive a recommendation for promotion compared to recruits who did not. Second, following Dolan et $\mathrm{al}^{33}$ and Mikulincer and Florian, ${ }^{18}$ we expected higher scores for functional and lower scores for dysfunctional coping styles in recruits who went on to receive a recommendation for promotion compared to recruits who did not. Third, based on Penley et al ${ }^{16}$ and Taylor and Stanton, ${ }^{17}$ we expected that functional coping styles would be associated with lower distress levels, while dysfunctional coping styles would be associated with elevated distress levels.

\section{Methods \\ Procedure}

Two different groups were assessed and compared in the present study, recruits who received a recommendation for promotion at the end of general basic training (ie, week 6) and recruits who did not and remained enlisted for the remainder of their military service. The group allocation was thus necessarily determined a posteriori. The survey was carried out with seven different military units (entire companies) on four training grounds in order to obtain a balance between combat and support troops. Psychological distress was measured during week 4 of basic training. Coping style in its conceptualization as a stable trait variable ${ }^{38}$ was measured during the first week of basic training. For both instances, participants were assembled company-wise at the end of a classroom lesson and had 20 minutes to complete a series of standardized questionnaires related to basic sociodemographic data and psychological distress coping styles. They received instructions on how to fill out the questionnaires by two trained officers of the Psychologic-Pedagogic Service of the Swiss Armed Forces, who were assisted by NCOs of the respective training ground. Missing cases were thus prevented and missing values kept to a minimum. Although the present analysis draws on a broader ongoing longitudinal study, the results reported here on psychological distress and coping styles have not been published, discussed, or presented elsewhere. Thus, the findings are novel.

\section{Sample}

Written informed consent was obtained from all participants. The Psychologic-Pedagogic Service and Armed Forces Personnel approved the study, which was performed in accordance with the principles laid down in the Declaration of Helsinki. A total of 675 male recruits (aged $=18-26$ years, $M=20.03$ years, standard deviation $=1.14$ ) of the Swiss Armed Forces were examined in a cross-sectional study during the first 4 weeks of basic military training. Of this sample, 100 were recommended for promotion by their superiors at the end of general basic training, while 575 were not and remained enlisted during the remainder of their service. Although some preenlistment psychometric data are also used in selecting future cadre (eg, IQ), they are used as exclusion rather than inclusion criteria and are thus of only low discriminatory power.

\section{Materials}

\section{Psychological distress}

Psychological distress was assessed using the Symptom Checklist-90-Revised (SCL-90-R, Pearson, San Antonio, TX, USA), a 90-item self-report symptom inventory designed to reflect psychological symptom patterns with a time reference of the past 7 days. ${ }^{39}$ Respondents are asked how much they have been bothered by issues such as faintness or dizziness, crying easily, feeling fearful, or having urges to break or smash things. Distress severity is rated on fivepoint rating scales with the anchor points 0 (= not at all) to 4 (= extremely); thus, higher scores reflect greater distress. The SCL-90-R is widely used and has been found to be a valid and reliable measure for psychological distress, both in English and in German. ${ }^{40}$ In our survey, the original nine dimensions of the SCL-90-R were reduced to the following five: Depression (13 items), Somatization (12 items), Anxiety (ten items), Interpersonal Sensitivity (nine items), and Hostility ( six items). The scales Obsessive-Compulsive, Psychoticism, and Paranoia were omitted so as to reduce respondent burden and because recruits with mental disorders on axes 1 and 2 of the DSM-IV are screened out during recruitment and declared unfit for service. The dimensions have internal consistencies ranging from $\alpha=0.77$ to 0.90 , and the inventory itself shows high concurrent validity with the Minnesota Multiphasic Personality Inventory. ${ }^{39}$

\section{Coping}

Coping styles were assessed with the Individual Coping Questionnaire (INCOPE-2), a 23-item questionnaire consisting of six subscales that reflect a variety of individual coping strategies for psychosocial stress. ${ }^{41}$ Items are rated on fivepoint rating scales with respect to the frequency with which each coping strategy is used in everyday life, with anchor points 0 (=never) and 4 (= always). The six subscales assess Rumination (four items; eg, intellectual brooding, feeling guilty; $\alpha=0.70$ ), Positive Self-Verbalization (three items; eg, humor, positive self-instruction; $\alpha=0.74$ ), Active Problem Solving (seven items; eg, active engagement, search for social support; $\alpha=0.71$ ), Expression of Negative Emotions 
(three items; eg, blaming comrades; $\alpha=0.73$ ), Negative Relaxation Strategies (three items; eg, alcohol consumption; $\alpha=0.75$ ), and Withdrawal/Avoidance (three items; eg, withdrawal of the stressful event; $\alpha=0.86$ ). The subscales have internal consistencies ranging from $\alpha=0.70$ to $\alpha=0.86$. Two single items measure the subjective Effectiveness of one's coping style and one's Satisfaction with it. The subscales Rumination, Expression of Negative Emotions, Negative Relaxation Strategies, and Withdrawal/Avoidance are aggregated to the dimension Dysfunctional Coping, while the subscales Positive Self-Verbalization and Active Problem Solving are aggregated to the dimension Functional Coping. Finally, a Total Score indicating positive coping is computed from all items. The dimensions have internal consistencies of $\alpha=0.71$ for Dysfunctional Coping, $\alpha=0.70$ for Functional Coping, and $\alpha=0.76$ for the Total Score, and validation studies have demonstrated satisfactory concurrent and predictive validity. ${ }^{41}$

\section{Statistical analysis}

The SCL-90-R dimensions and INCOPE-2 scales were tested for normality and homogenous variances. Both the Kolmogorov-Smirnov test and the Skewness and Kurtosis test revealed significant departures from normal distributions for all scales and dimensions in both groups. Levene tests revealed that all SCL-90 dimensions were heteroscedastic, while INCOPE-2 scales exhibited both heterogeneous and homogenous variances. Consequently, two-tailed MannWhitney tests were performed to determine differences in psychological distress and coping styles between the two groups, and two-tailed Spearman rank correlations were performed to test the association between distress levels and coping styles. Missing values were excluded case-wise, and the nominal level of significance was set at $\alpha<0.05$. All statistical computations were performed with SPSS ${ }^{\circledR} 23.0$ (IBM Corporation, Armonk, NY, USA) for Windows ${ }^{\circledR}$ and $\mathrm{G}^{*}$ Power $^{\circledR} 3.1 .9$ (University of Düsseldorf, Düsseldorf, Germany). ${ }^{42}$

\section{Results}

\section{Group differences in psychological distress}

The prospective cadre group reported significantly lower Depression scores $(\mathrm{Mdn}=0.13)$ than the recruits who remained enlisted $(\mathrm{Mdn}=0.25), U=25,034.5, P<0.05$, $r=0.08$. Likewise, the prospective cadre group reported significantly lower Hostility scores $(\mathrm{Mdn}=0.00)$ than the other recruits ( $\mathrm{Mdn}=0.17), U=24,074.0, P<0.01, r=0.10$. Post hoc power computations for the Depression analysis revealed an achieved power of 0.72 and for the Hostility analysis an achieved power of 0.89 .

No statistically significant differences between the two groups were observed for the dimensions Somatization, Anxiety, and Interpersonal Sensitivity, with $26,270.0 \leq U \leq 27,278.5$.

\section{Group differences in coping styles}

The prospective cadre group reported significantly higher scores for Active Problem Solving $(\mathrm{Mdn}=2.40)$ than the other recruits ( $\mathrm{Mdn}=2.20), U=26,051.5, P<0.05, r=0.09$. They furthermore reported higher scores for Effectiveness of coping $(\mathrm{Mdn}=3.00)$ than the other recruits $(\mathrm{Mdn}=3.00)$, $U=25,926.5, P<0.05, r=0.09$. Although the cadre group reported higher Total Scores $(\mathrm{Mdn}=0.98)$ than the other recruits $(\mathrm{Mdn}=0.79)$, this difference just failed to reach a significant level, $U=26,778.0, P=0.054, r=0.07$. Post hoc power computations for the Active Problem Solving analysis revealed an achieved power of 0.33 and for the Effectiveness analysis an achieved power of 0.71 .

No statistically significant differences between the two groups were observed for the dimensions Dysfunctional Coping, Functional Coping, Rumination, Positive SelfVerbalization, Expression of Negative Emotions, Negative Relaxation Strategies, and Withdrawal/Avoidance, or for the single item Satisfaction, with 26,977.5 $\leq U \leq 30,219.5$.

\section{Association between distress and coping}

All correlation coefficients between dimensions of psychological distress and functional and dysfunctional coping styles are reported in Table 1 . The pattern of results showed that functional coping correlated negatively and significantly with all distress scales, whereas dysfunctional coping correlated positively and significantly with all distress scales, although less strongly. Post hoc power computations revealed that the achieved power ranged from 0.83 for the lowest correlation coefficient to 0.95 for the highest correlation coefficient.

Table I Correlation between coping style and distress levels

\begin{tabular}{llllll}
\hline & Depression & Somatization & Anxiety & Interpersonal sensitivity & Hostility \\
\hline Functional coping & $-0.19^{*}$ & $-0.13^{*}$ & $-0.19^{*}$ & $-0.15^{*}$ & $-0.22^{*}$ \\
Dysfunctional coping & $0.17^{*}$ & $0.10^{*}$ & $0.18^{*}$ & $0.15^{*}$ & $0.17^{*}$ \\
\hline
\end{tabular}

Notes: $* p<0.01$, $* * p<0.05$. 


\section{Discussion}

The key findings of the present study were that the prospective cadre exhibited lower levels of depression and hostility and reported more active and effective coping than the nonprospective cadre recruits. The present data add to the current literature in an important way in that we were able to show that cadre selection as an experts' rating was mirrored by favorable self-rated psychological processes.

Three hypotheses were formulated and each of these is considered in turn. With the first hypothesis, we expected lower psychological distress in prospective cadre compared to nonprospective cadre, and this was partially confirmed. Specifically, prospective cadre had lower depression and hostility scores. This pattern of results matched those of Westman ${ }^{13}$ and Maddi et al, ${ }^{14}$ in as much as higher stress resistance went along with the successful passing of demanding training courses, eg, basic military training. However, the normative expectation that future military leaders experience less stress than their enlisted comrades has, to the best of our knowledge, never been empirically validated. The present data thus add to the current literature in that we were able to confirm that recruits who impress their superior officers as qualified for promotion do not only exhibit stress-moderating traits such as hardiness but also report less actual (subjective) distress itself. In addition, the results suggest that the superior officers in charge of recruits attach' special importance to social skills and prosocial behavior in their assessment of the recruit's leadership qualities. One of the constituent elements of depression is a lack of social activity, ${ }^{43}$ while hostility is characterized by socially antagonistic behavior. In both cases, social behavior is affected in a detrimental way. Low hostility has thus not surprisingly been shown to be closely related to leadership effectiveness in military settings. ${ }^{22}$ Similarly, military culture may counterintuitively be characterized by a low level of masculinity. ${ }^{44}$ Stereotypical masculine values such as competitiveness, materialism, ambition, assertiveness, and personal power are consequently held in low esteem in a military setting. Recruits who impress their superiors as potential cadre seem to do so by exhibiting a proactive element of sociability and by lacking a resentful or vindictive streak. This is consistent with the Swiss Armed Forces' focus on social skills when selecting the cadre, as well as classic leadership theory and its emphasis on people orientation or consideration. ${ }^{19}$ These prosocial attitudes and behaviors are not merely of ethical importance; however; they also have their usefulness. Devries postulates that prosocial conduct, eg, affiliative behavior, can provide a coping mechanism with respect to stressful social conditions that arise during the establishment of social rank relationships. ${ }^{48}$
This suggests that the prospective cadre's prosocial behavior not only mitigates the potentially negative group dynamics that accompany their leadership emergence but also allows them to cope with their own stress resulting from the concomitant social dynamics.

Our second hypothesis was that more functional and less dysfunctional coping would characterize prospective cadre compared to nonprospective cadre, and this too was partially confirmed. Recruits with a recommendation for promotion at the end of general basic training engaged in more active problem solving, reported more effective coping, and exhibited a tendency to cope more favorably in general. This concurs with findings of both Pratch and Jacobowitz ${ }^{35}$ and Mikulincer and Florian, ${ }^{18}$ who found that effective junior military leaders engaged less in emotion-oriented and more in problem-oriented coping, with the latter coping style being associated with a more self-efficacious mind set. In view of the results from our first two hypotheses, the combination of low hostility and high sense of coping effectiveness that we found in prospective cadre candidates would also suggest a lower risk of developing psychopathological symptoms after a potentially traumatic experience: a prospective follow-up study with firefighters was able to show that high levels of hostility and low levels of self-efficacy at baseline accounted for half of the variance in posttraumatic stress disorder symptoms after 2 years on the job. ${ }^{49}$ It also predicted a significant increase in measures of posttraumatic stress disorder symptoms, depression, anxiety, and general psychological morbidity.

Our third hypothesis was that functional coping strategies would be associated with lower distress scores and that dysfunctional coping would be associated with higher distress scores. Our data confirm this and corroborate studies by Repetti et al ${ }^{50}$ and Taylor and Stanton, ${ }^{17}$ who were able to show that psychological distress was related to a lack of adequate coping resources. It is noteworthy that functional coping exhibited a greater association with distress than dysfunctional coping. This suggests that it is not so much a lack of dysfunctional coping that contributes to lower psychological distress but rather the presence of functional coping.

Despite the novelty of the results, several limitations warrant against their overgeneralization. First, the sample consisted of only male recruits; accordingly, it is not clear to what extent the present data are also applicable to female recruits. Second, no objective measures were made; it would have increased the validity to investigate to what extent selfratings of psychological distress and coping strategies were consistent (or inconsistent) with a nonpharmacological stress test such as the Trier Social Stress Test. ${ }^{51}$ Third, the analyses 
were cross-sectional and correlational; this allows us to draw only conjectural conclusions on the causality of the association between psychological distress and coping styles. Fourth, the effect sizes of the differences in psychological distress and coping styles were small, despite the generally adequate power that was achieved. However, the dichotomous classification of recruits into the groups "promotion" versus "no promotion" is rather crude and ignores many additionally relevant characteristics of the participants, such as social support and life events. Insofar, the small effect sizes are explicable. Finally, it would have been interesting to follow-up both groups of recruits with regard to their military performance in order to gain further insight into the predictive validity of such assessments.

In view of these limitations, further research should focus on a more sophisticated classification of participants and supplement the assessed data with objective measures of stress, eg, salivary cortisol. In addition, a further breakdown of the superiors' appraisal of the recruits' aptitude for promotion would allow the identification of those criteria that are pivotal in the eyes of the superior officers. A promising instrument for future research is the Military Training Mental Toughness Inventory, which is based on the Mental Toughness Inventory. ${ }^{52}$ The latter in turn was conceptualized within Gray and McNaughton's neuropsychological framework of the revised Reward Sensitivity Theory, which aims to explain how goal-directed behavior is maintained under stress. Military training mental toughness may moderate the relationship between psychological distress and performance in basic training on the one hand, and reveal a relationship with certain coping styles on the other. Finally, future studies should be longitudinal, thus allowing to draw firm conclusions about the causality of the associations.

\section{Conclusion}

The pattern of results suggests that prospective cadre group is able to maintain their social agency even under elevated physical and psychological stress when compared to the members of their cohort who were not recommended for promotion and remained enlisted. The capacity to cope with psychosocial stress and remain influential in a social group goes hand in hand with lower levels of socially adverse stress responses, possibly acting as a buffer. This specific pattern of coping style and stress response has the additional benefit of facilitating the recruit's emergence as a leader by allowing him to develop and utilize his leadership skills under stress, because research on transformational leadership has shown that prosocial interpersonal skills such as individualized consideration are crucial factors in effective leadership..$^{53}$

\section{Acknowledgment}

The authors thank Nick Emler (University of Surrey, Surrey UK) for proofreading the manuscript.

\section{Disclosure}

The authors report no conflicts of interest in this work.

\section{References}

1. Smith TC, Wingard DL, Ryan MA, Kritz-Silverstein D, Slymen DJ, Sallis JF. PTSD prevalence, associated exposures, and functional health outcomes in a large, population-based military cohort. Public Health Rep. 2009;124(1):90-102.

2. Hoge CW, Castro CA, Messer SC, McGurk D, Cotting DI, Koffman RL. Combat Duty in Iraq and Afghanistan, Mental Health Problems, and Barriers to Care. N Engl J Med. 2004;351(1):13-22.

3. Orasanu JM, Backer P. Stress and military performance. In: Driskell J, Salas E, editors. Stress and Human Performance. Mahwah, NJ: Lawrence Erlbaum Associates; 1996:89-126.

4. Bernton E, Hoover D, Galloway R, Popp K. Adaptation to chronic stress in military trainees. Adrenal androgens, testosterone, glucocorticoids, IGF-1, and immune function. Ann N Y Acad Sci. 1995;774(1): 217-231.

5. Clow A, Edwards S, Owen G, et al. Post-awakening cortisol secretion during basic military training. Int J Psychophysiol. 2006;60(1):88-94.

6. Hellhammer DH, Buchtal J, Gutberlet I, Kirschbaum C. Social hierarchy and adrenocortical stress reactivity in men. Psychoneuroendocrinology. 1997;22(8):643-650.

7. Taylor MK, Sausen KP, Potterat EG, et al. Stressful military training: endocrine reactivity, performance, and psychological impact. Aviat Space Environ Med. 2007;78(12):1143-1149.

8. Booth CK, Probert B, Forbes-Ewan C, Coad RA. Australian army recruits in training display symptoms of overtraining. Mil Med. 2006; 171(11):1059-1064.

9. Martin PD, Williamson DA, Alfonso AJ, Ryan DH. Psychological adjustment during army basic training. Mil Med. 2006;171(2):157-160.

10. La Marca R, Boesch M, Trulec-Sefidan S, et al. A decrease in perceived social support during military service is associated with a concomitant increase in baseline and decrease in stress reactivity levels of salivary alpha-amylase. Eur J Psychotraumatology. 2012;(Suppl 1):109.

11. Lerew DR, Schmidt NB, Jackson RJ. Evaluation of psychological risk factors: prospective prediction of psychopathology during basic training. Mil Med. 1999;164(7):509.

12. Clemons EP. Monitoring anxiety levels and coping skills among military recruits. Mil Med. 1996;161(1):18-21.

13. Westman M. The relationship between stress and performance: the moderating effect of hardiness. Hum Perform. 1990;3(3):141-155.

14. Maddi SR, Harvey RH, Resurreccion R, Giatras CD, Raganold S. Hardiness as a performance enhancer in firefighters. Int J Fire Service Leadership Management. 2007;1(2):3-9.

15. Vogt DS, Rizvi SL, Shipherd JC, Resick PA. Longitudinal investigation of reciprocal relationship between stress reactions and hardiness. Pers Soc Psychol Bull. 2008;34(1):61-73.

16. Penley JA, Tomaka J, Wiebe JS. The association of coping to physical and psychological health outcomes: a meta-analytic review. J Behav Med. 2002;25(6):551-603.

17. Taylor SE, Stanton AL. Coping resources, coping processes, and mental health. Annu Rev Clin Psychol. 2007;3(1):377-401.

18. Mikulincer M, Florian V. Appraisal of and coping with a real-life stressful situation: the contribution of attachment styles. Pers Soc Psychol Bull. 1995;21(4):406-414.

19. Bass BM. The Bass Handbook of Leadership: Theory, Research, and Managerial Applications. New York, NY: Free Press; 2008.

20. Hogan R, Curphy GJ, Hogan J. What we know about leadership. Effectiveness and personality. Am Psychol. 1994;49(6):493-504. 
21. Koolhaas JM, Korte SM, De Boer SF, et al. Coping styles in animals: current status in behavior and stress-physiology. Neurosci Biobehav Rev. 1999;23(7):925-935.

22. Judge TA, Bono JE, Ilies R, Gerhardt MW. Personality and leadership: a qualitative and quantitative review. J Appl Psychol. 2002;87(4): 765-780.

23. Hogan J, Holland B. Using theory to evaluate personality and job-performance relations: a socioanalytic perspective. J Appl Psychol. 2003;88(1):100-112.

24. Salgado JF. Predicting job performance using FFM and non-FFM personality measures. J Occup Organ Psy. 2003;76(3):323-346.

25. McBurnett K, Raine A, Stouthamer-Loeber M, et al. Mood and hormone responses to psychological challenge in adolescent males with conduct problems. Biol Psychiatry. 2005;57(10):1109-1116.

26. van Bokhoven I, van Goozen SH, van Engeland H, et al. Salivary cortisol and aggression in a population-based longitudinal study of adolescent males. J Neural Transm (Vienna). 2005;112(8):1083-1096.

27. Mohr G, Wolfram HJ. Stress among managers: the importance of dynamic tasks, predictability, and social support in unpredictable times. J Occup Health Psychol. 2010;15(2):167-179.

28. Fiedler F, Potter E, McGuire M. Stress and effective leadership decisions. In: Heller FA, editor. Decision Making and Leadership. Cambridge, UK: Cambridge University Press; 1992:47-57.

29. Bartone PT. Resilience under military operational stress: can leaders influence hardiness? Milit Psychol. 2006;18(Suppl):S131-S148.

30. Bliese PD. Social climates: drivers of soldier well-being and resilience. In: Adler AB, Castro CA, Britt TW, editors. Military Life: The Psychology of Serving in Peace and Combat: Operational Stress. Vol 2. Westport, CT: Praeger Security International; 2006:213-234.

31. Castro CA, McGurk D. The intensity of combat and behavioral health status. Traumatology. 2007;13(4):6-23.

32. Gold MA, Friedman SB. Cadet basic training: an ethnographic study of stress and coping. Mil Med. 2000;165(2):147-152.

33. Dolan C, Huffman A, Adler A, Wright K, Thomas J, Castro C. Coping with the stress of a military deployment: psychological and physical health. Paper presented at the 22nd Annual Stress and Anxiety Research International Conference; 2001; Mallorca, Spain.

34. Carroll L. Passive coping strategies. In: Gellman MD, Turner JR, editors. Encyclopedia of Behavioral Medicine. New York, NY: Springer; 2013:1442.

35. Pratch L, Jacobowitz J. Gender, motivation, and coping in the evaluation of leadership effectiveness. Consult Psychol J. 1996;48(4):203-220.

36. Carroll L. Active coping. In: Gellman MD, Turner JR, editors. Encyclopedia of Behavioral Medicine. New York, NY: Springer; 2013:21.

37. Bartone PT, Snook SA, Tremble TR. Cognitive and personality predictors of leader performance in West Point cadets. Mil Psychol. 2002; 14(4):321-338.

38. Beutler LE, Moos RH, Lane G. Coping, treatment planning, and treatment outcome: discussion. J Clin Psychol. 2003;59(10):1151-1167.
39. Derogatis LR. SCL-90-R, Administration, Scoring and Procedures Manual II for the Revised Version and Other Instruments of the Psychopathology Rating Scale Series. Baltimore, MD: Clinical Psychometric Research; 1992.

40. Schmitz N, Hartkamp N, Kiuse J, Franke GH, Reister G, Tress W. The Symptom Check-List-90-R (SCL-90-R): a German validation study. Qual Life Res. 2000;9(2):185-193.

41. Bodenmann G. Stress und Coping bei Paaren [Stress and Coping in Couples]. Göttingen, Germany: Hogrefe; 2000.

42. Faul F, Erdfelder E, Lang AG, Buchner A. G*Power 3: a flexible statistical power analysis program for the social, behavioral, and biomedical sciences. Behav Res Methods. 2007;39(2):175-191.

43. American Psychiatric Association. Diagnostic and Statistical Manual of Mental Disorders. 5th ed. Arlington, VA: American Psychiatric Publishing; 2013.

44. Hofstede GH. Culture's Consequences: Comparing Values, Behaviors, Institutions, and Organizations Across Nations. 2nd ed. Thousand Oaks, CA: Sage Publications; 2001.

45. Page J. Culture and the Profession of Arms in the 21st century: An Application of Hofstede's Theory within the North Atlantic Treaty Organisation (NATO) and the Partnership for Peace (PfP) Military Cultures Fort Lauderdale, FL: Nova Southeastern University; 2003.

46. Soeters JL. Value orientations in military academies: a thirteen country study. Armed Forces Soc. 1997;24(1):7-32.

47. Soeters JL, Recht R. Convergence or divergence in the multinational classroom? Experiences from the military. Int J Intercult Relat. 2001; 25(4):423-440.

48. Devries A, Glasper E, Detillion C. Social modulation of stress responses. Physiol Behav. 2003;79(3):399-407.

49. Heinrichs M, Wagner D, Schoch W, Soravia LM, Hellhammer DH, Ehlert U. Predicting posttraumatic stress symptoms from pretraumatic risk factors: a 2-year prospective follow-up study in firefighters. $\mathrm{Am}$ J Psychiatry. 2005;162(12):2276-2286.

50. Repetti RL, Taylor SE, Seeman TE. Risky families: family social environments and the mental and physical health of offspring. Psychol Bull. 2002;128(2):330-366.

51. Kirschbaum C, Pirke KM, Hellhammer DH. The "Trier Social Stress Test" - a tool for investigating psychobiological stress responses in a laboratory setting. Neuropsychobiology. 1993;28(1-2):76-81.

52. Arthur CA, Fitzwater J, Hardy L, Beattie S, Bell J. Development and validation of a military training mental toughness inventory. Mil Psychol. 2015;27(4):232.

53. Bass B, Avolio B. Improving Organizational Effectiveness Through Transformational Leadership. Thousand Oaks, CA: Sage Publications; 1994.
Neuropsychiatric Disease and Treatment

\section{Publish your work in this journal}

Neuropsychiatric Disease and Treatment is an international, peerreviewed journal of clinical therapeutics and pharmacology focusing on concise rapid reporting of clinical or pre-clinical studies on a range of neuropsychiatric and neurological disorders. This journa is indexed on PubMed Central, the 'PsycINFO' database and CAS,

\section{Dovepress}

and is the official journal of The International Neuropsychiatric Association (INA). The manuscript management system is completely online and includes a very quick and fair peer-review system, which is all easy to use. Visit http://www.dovepress.com/testimonials.php to read real quotes from published authors. 